\title{
Propagation and scattering of TE surface plasmon polaritons on interface between two dielectrics
}

\author{
Tomáš Váry, Peter Markoš \\ Department of Physics FEI STU, Ilkovičova 3, 81219 Bratislava, Slovakia
}

\begin{abstract}
We study the TE polarized electromagnetic surface wave propagating along the interface between materials with positive and negative magnetic permeability. Contrary to the TM polarized surface wave, the TE surface wave exhibits almost no radiation losses when scattered at semi-infinite dielectric interfaces.
\end{abstract}

Key words: surface plasmons, metamaterials, planar optics

PACS: 42.25.Bs, 73.20.Mf

\section{Introduction}

Electromagnetic surface waves (SW) [1] provides us with new possibilities of engineering photonic devices and optical applications. Due to their purely twodimensional character of propagation they are predetermined to be used in the field of planar optics. The main constrain in the application of electromagnetic surface waves are radiation losses due to the scattering of the wave at surface inhomogeneities. The transmission of the surface plasmon through a single permittivity step [2, 3] might be accompanied with the excitation of the broad spectra of plane waves. The surface wave can lose more than $40 \%$ of its energy. This instability of the surface wave represents a strong constrain in the development of a surface wave optics since constructing optical devices based on multiple interfaces is rather ineffective.

The TM surface waves are excited at the metal dielectric interface, where the dielectric permittivity $\epsilon$ changes its sign [5, 6]. The construction of new metamaterials with negative magnetic permeability [7] opens a possibility to study also TE surface waves [8, 6]. In this letter, we show that radiation losses due to the propagation of the TE waves at the surface of negative permeability material are three orders of magnitude smaller than that for the TM wave at the metal-dielectric surface.

Email address: peter.markos@stuba.sk (Peter Markoš) Preprint submitted to Elsevier

\section{TE polarized surface plasmon polariton}

The TE polarized SW propagates along the interface of the negative-permeability material (NPM), located in the $x y$ plane and decreases exponentially in the $z$ direction as $\exp \left(-\kappa_{d} z\right)$ for $z>0$ (dielectric) and $\exp \left(+\kappa_{m} z\right)$ for $z<0$ (NPM). The dispersion relation for the TE SW is

$$
\frac{\kappa_{d}}{\mu_{d}}+\frac{\kappa_{m}}{\mu_{m}}=0
$$

An explicit form of the components of wave vectors read

$$
\kappa_{d}^{2}=k_{0}^{2} \mu_{d}^{2} \frac{\epsilon_{d} \mu_{m}-\epsilon_{m} \mu_{d}}{\mu_{m}^{2}-\mu_{d}^{2}}, \quad \kappa_{m}^{2}=\frac{\mu_{m}^{2}}{\mu_{d}^{2}} \kappa_{d}^{2}, \quad k_{\|}^{2}=\frac{\mu_{m}}{\mu_{d}} \kappa_{d}^{2} .
$$

Here, $k_{0}=\omega / c$ and $\overrightarrow{k_{\|}}=\left(k_{x}, k_{y}\right)$ is the projection of the wave vector $\vec{k}$ into the (xy) plane. Field components of the surface wave are

$$
\begin{aligned}
& \left.\begin{array}{l}
\vec{e}=\mathcal{N}_{0} e^{-\kappa_{d} z}\left(\frac{-k_{y}}{k_{\|}}, \frac{k_{x}}{k_{\|}}, 0\right) f(x, y) \\
\vec{h}=\frac{\mathcal{N}_{0}}{k_{0} z_{0}} e^{-\kappa_{d} z}\left(-\frac{k_{x} k_{z}}{k_{\|}},-\frac{k_{y} k_{z}}{k_{\|}}, k_{\|}\right) f(x, y)
\end{array}\right\} z \geq 0 \\
& \left.\begin{array}{l}
\vec{e}=\mathcal{N}_{0} e^{k_{m} z}\left(\frac{-k_{y}}{k_{\|}}, \frac{k_{x}}{k_{\|}}, 0\right) f(x, y) \\
\vec{h}=\frac{N_{0}}{k_{0} z_{0} \mu_{m}} e^{k_{m} z}\left(-\frac{k_{x} k_{z}}{k_{\|}},-\frac{k_{y} k_{z}}{k_{\|}}, k_{\|}\right) f(x, y)
\end{array}\right\} z<0,
\end{aligned}
$$

where $f(x, y)=e^{i\left(k_{x} x+k_{y} y\right)}$ and $z_{0}=\sqrt{\mu_{0} / \epsilon_{0}}$. The normalization coefficient $\mathcal{N}_{0}$ will be specified later. In what follows, we consider the permeability of dielectrics $\mu_{d} \equiv 1$, and frequency dependent permeability of NPM, $\mu_{m}=$ $\mu_{2}(\omega)$.

December 4, 2018 
Consider now the NPM covered by two different dielectrics. The interface between the two dielectrics is located in the $x=0$ plane, and creates the permittivity step: $\epsilon_{d}(x)=\epsilon_{1}(x<0)$ and $=\epsilon_{2}(x>0)$. Our aim is to calculate the transmission and reflection coefficients of the TE SW propagating through this permittivity discontinuity.

To compare the obtained data with those for the TM SW at the metal-dielectric interface, we consider the lossles Drude formula for the permittivity of metal $\epsilon_{\text {metal }}(\omega)=1-\omega_{p}^{2} / \omega^{2}$ and the same dispersion relation for the permeability of NPM:

$$
\mu_{m}(\omega)=1-\frac{\omega_{0}^{2}}{\omega^{2}}
$$

with $\omega_{0}=\omega_{p}$. Also, we assume $\mu_{\text {metal }}=1$ (nonmagnetic) and $\epsilon_{\mathrm{NPM}}=1$ for our NPM. This choice of parameters give us clear confrontation between behavior of the TM and TE SW on the surface of metal and of NPM. The exact form of the frequency dependence is not important since only single frequency $\omega$ will be considered.

\section{The method}

For the calculation of the scattering parameters we apply a modified method of Oulton et al. [3] based on the scattering matrix approach. The details of the method are given elsewhere [4]. The surface wave is coming from the left and scatters on the interface between the two dielectrics. Owing to the nonhomogeneous $z$ dependence of electric and magnetic field of the surface wave, the matching of the tangential components of the fields at the $x=0$ interface is possible only with the assistance of plane waves. We consider $N$ plane waves with the same frequency $\omega$ and different $z$ components of the wave vector $k_{z}=k_{\max } \alpha$, $\alpha=1, \ldots N$. The continuity equations for the $y$ component of electric and $z$ component of magnetic field are

$$
\begin{aligned}
\left(A_{i 0}+\bar{A}_{i 0}\right) e_{i} & +\sum_{\alpha}^{N}\left[A_{\alpha}+\bar{A}_{\alpha}\right] E_{i \alpha} \\
& =\left(A_{j 0}+\bar{A}_{j 0}\right) e_{2}+\sum_{\alpha}^{N}\left[A_{j \alpha}+\bar{A}_{j \alpha}\right] E_{j \alpha}, \\
\left(A_{i 0}-\bar{A}_{i 0}\right) h_{i} & +\sum_{\alpha}^{N}\left[A_{\alpha}-\bar{A}_{\alpha}\right] H_{i \alpha} \\
& =\left(A_{j 0}-\bar{A}_{j 0}\right) h_{j}+\sum_{\alpha}^{N}\left[A_{j \alpha}-\bar{A}_{j \alpha}\right] H_{j \alpha} .
\end{aligned}
$$

Indices $i, j=1,2$ correspond to the dielectric medium. $A$ and $\bar{A}$ are the amplitudes of the wave propagating to the left (right) in the first media. Index 0 indicates SW. The explicit form of the $y$-component of the electric and $z$-component of the magnetic fields is

$$
E_{i \alpha}=\mathcal{N}_{i \alpha} \frac{k_{x, i \alpha}}{k_{\|_{\alpha}}} \times\left\{\begin{array}{cc}
{\left[-e^{-i k_{z \alpha} z}+r_{i \alpha} e^{i k_{z \alpha}} z\right]} & z>0 \\
{\left[-t_{i \alpha} e^{-i k_{z m i \alpha} z}\right]} & z<0,
\end{array}\right.
$$

and

$$
H_{j \alpha}=\frac{\mathcal{N}_{j \alpha}}{z_{0} k_{0}} k_{\|_{\alpha}} \times\left\{\begin{array}{cc}
{\left[-e^{-i k_{z j \alpha} z}+r_{j \alpha} e^{i k_{z j \alpha} z}\right]} & z>0 \\
{\left[-t_{j \alpha} e^{-i k_{z m j \alpha} z}\right] / \mu_{m}} & z<0 .
\end{array}\right.
$$

$t_{i \alpha}=\left(1-r_{i \alpha}\right)$ and $r_{i \alpha}=\left(k_{z m i \alpha}-k_{z \alpha} \mu_{m}\right) /\left(k_{z m i \alpha}+k_{z i \alpha} \mu_{m}\right)$ are transmission and reflection amplitudes for the plane wave $\alpha$ incident at the interface of $i$ th dielectric and NPM.

In what follows, we use the matrix

$$
C_{\alpha \beta}^{i j}=\int_{-\infty}^{\infty} E_{i \alpha} H_{j \beta} d y d z .
$$

The requirement $C_{\alpha \beta}^{i i}=\delta_{\alpha \beta}$ determines the normalization constants $\mathcal{N}_{i \alpha}$. With the use of integrals (8) we rewrite (5) to the system of linear equations

$$
\begin{aligned}
& A_{2}+\bar{A}_{2}=\left[A_{1}+\bar{A}_{1}\right] C^{T}, \\
& A_{1}-\bar{A}_{1}=\left[A_{2}-\bar{A}_{2}\right] C,
\end{aligned}
$$

which can be rewritten into the form

$$
\left(\begin{array}{c}
A_{2} \\
\bar{A}_{1}
\end{array}\right)=S\left(\begin{array}{c}
\bar{A}_{2} \\
A_{1}
\end{array}\right)=\left(\begin{array}{ll}
S_{11} & S_{12} \\
S_{21} & S_{22}
\end{array}\right)\left(\begin{array}{c}
\bar{A}_{2} \\
A_{1}
\end{array}\right) .
$$

The matrix $S$ is a $2(N+1) \times 2(N+1)$ scattering matrix. The transmission and reflection coefficients for the SW incident from left media are $T=\left|S_{12}(0,0)\right|^{2}$ and $R=\left|S_{22}(0,0)\right|^{2}$. The radiation losses are given by the relation $S=\sum_{\alpha}^{\prime}\left(\left|S_{12}(\alpha, 0)\right|^{2}+\left|S_{22}(\alpha, 0)\right|^{2}\right)$ where the summation is over all plane waves with real component $k_{x}$. Typically $N=100-200$ plane waves are used in our analysis.

\section{Results}

\subsection{Transmission, reflection and Scattering losses}

Figure 1 shows the transmission and the reflection coefficients for the TE surface wave. The permeability of the NPM is $\mu_{m}=-17.9$. As expected, the transmission decreases and the reflection rises with increasing permittivity step between dielectric media. Radiative losses $S$ are negligibly small, $S \sim 10^{-4}$, even for $\epsilon_{2} / \epsilon_{1}=10$. This is in contrast with similar data for the

(5) TM surface wave propagating along the metal dielectric interface [3, 4] shown in Fig. 2] The permittivity of metal is $\epsilon_{\text {metal }}=-17.9$. We see that scattering losses are $10^{-3} \times$ smaller for the TE wave than for the TM one.

\subsection{Continuity of fields at the interface}

To test the accuracy of the method, we plot in Fig. 3 tangential components of electric, and magnetic, fields, given by Eqs. (5), along the $x=0$ plane on both sides of the interface for the permittivity step $\epsilon_{2} / \epsilon_{1}=5$. As can be seen, both fields are well matched on the interface. 


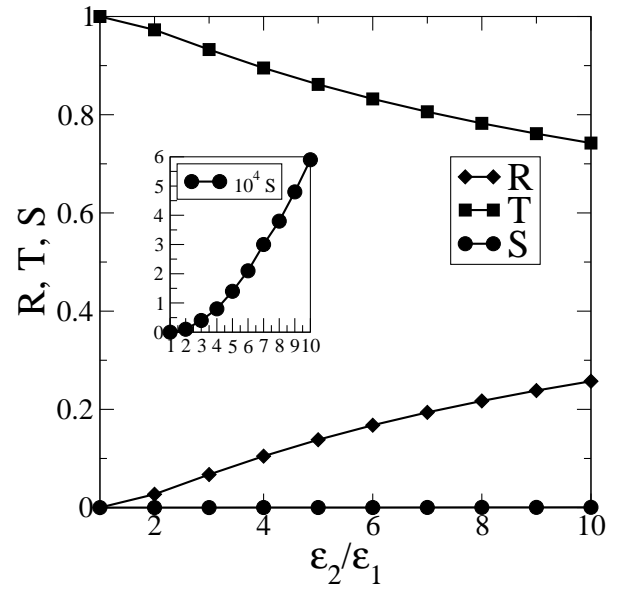

Figure 1: Transmission $T$, reflection $R$ and scattering losses $S$ for the TE polarized surface waves propagating along the NPM surface The negative permeability $\mu_{\mathrm{NPM}}=-17.9$. Inset shows radiative losses $S$.

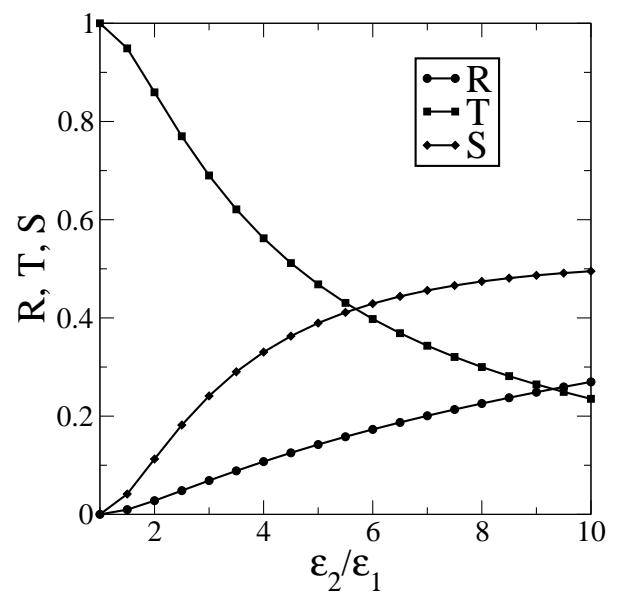

Figure 2: Transmission $T$, reflection $R$ and scattering losses $S$ as a function of permittivity step $\epsilon_{2} / \epsilon_{1}$ for the TM polarized surface waves propagating at the metal-dielectric interface. The permittivity of metal $\epsilon_{\text {metal }}=-17.9$.

\subsection{Oblique angle incidence}

In Fig. 4 we plot the dependence of the transmission, reflection and scattering losses on the angle of incidence. The refraction angle follows the modified Snell's law for TE polarized surface plasmons, which for our simple case has form

$$
\sin \theta_{2}=\sin \theta_{1} \sqrt{\frac{\mu \epsilon_{2}-1}{\mu \epsilon_{1}-1}} .
$$

The frequency dependence of the refraction angle $\theta_{2}$, given by the permittivity $\mu_{m}(\omega)$. shifts $\theta_{2}$ (compared
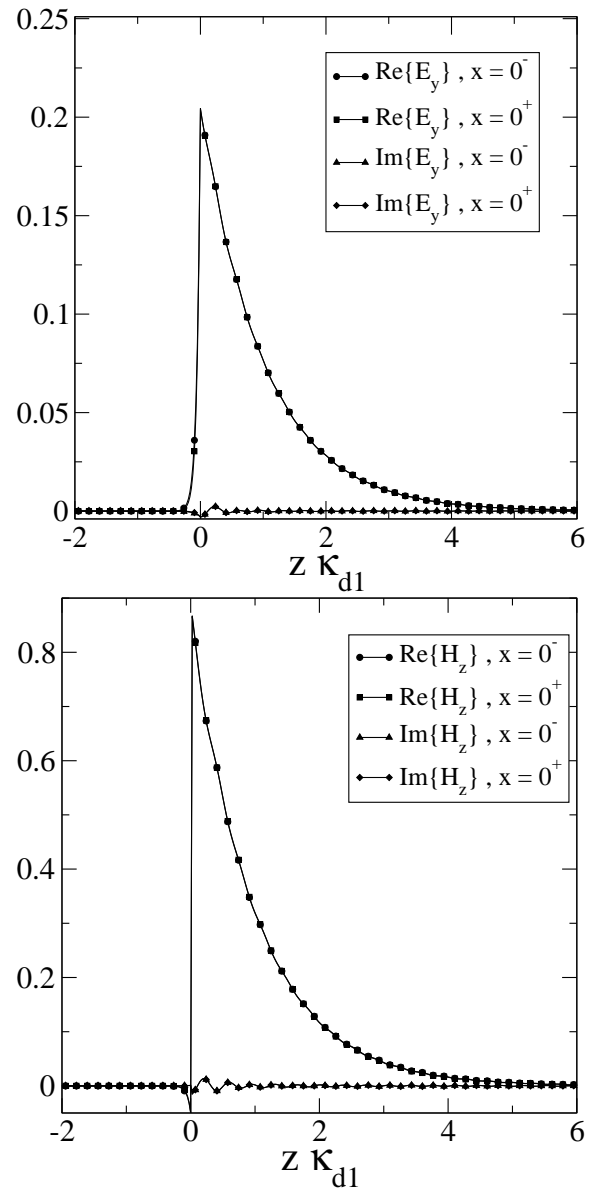

Figure 3: The test of the continuity of the electric (top) and magnetic (bottom) fields along the interface $x=0$. Permittivity step $\epsilon_{2} / \epsilon_{1}=5$. $\kappa_{d 1}$ is given by Eq. (2).

to the plane waves' refraction angle between similar dielectric media) to lower values for the SW incident from lower permittivity medium. Also, for SW incident from the media with higher permittivity, the critical angle for the surface waves is larger than that for the plane wave. Top panel of Fig. 4 shows the case for the incidence from optically less dense medium. As is expected, with the increasing angle of the incidence the transmission monotonously decreases and more significant part of the energy is reflected in the form of surface wave. The scattering losses are negligible for the entire interval of incident angles. Bottom panel shows the scattering of the surface wave incident from the medium with higher permittivity. The transmission vanishes when approaching the critical angle given by (11) for $\sin \theta_{2}=1$. Naturally, reflection must increase to one, as passing the critical angle. 

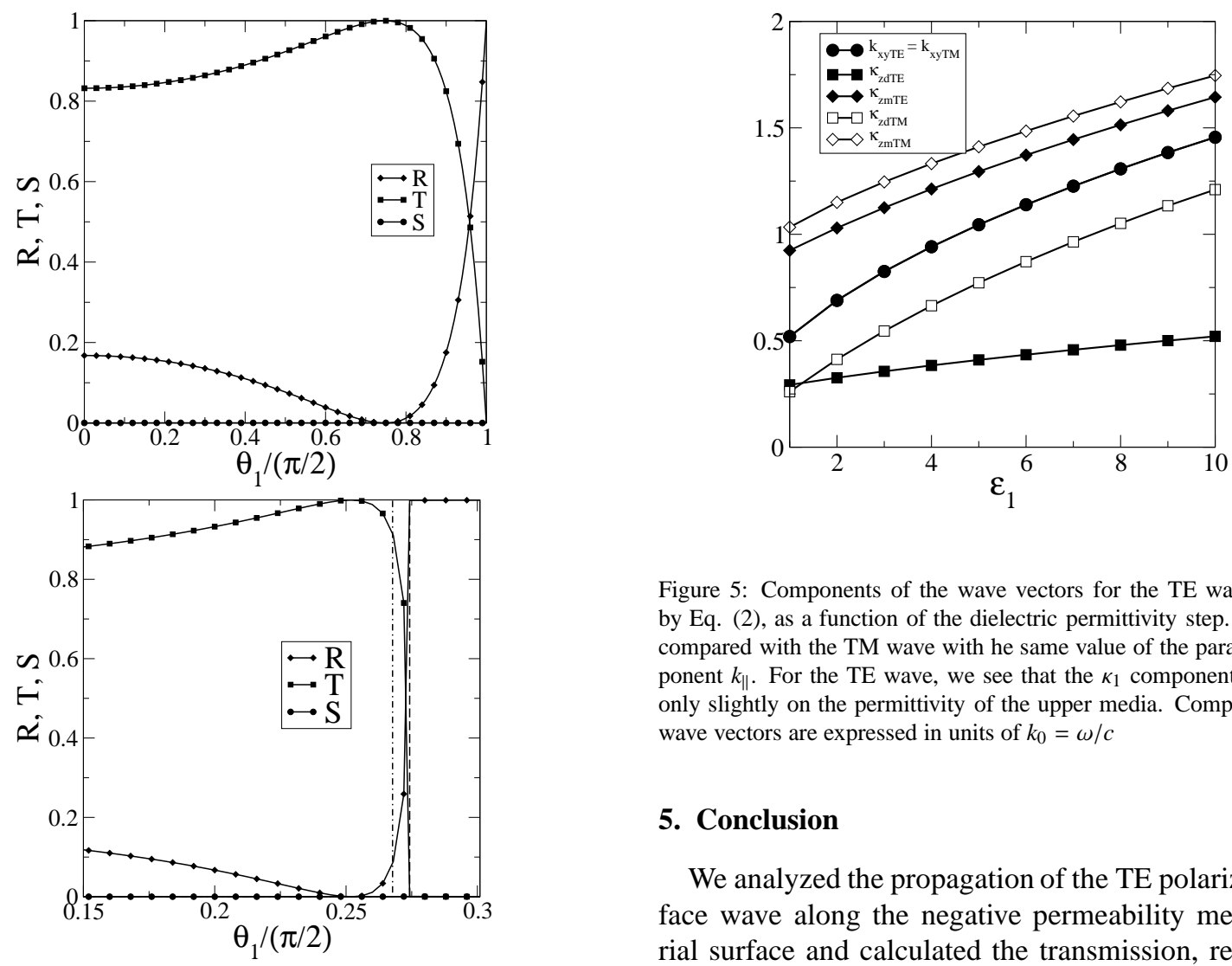

Figure 5: Components of the wave vectors for the TE wave, given by Eq. (2), as a function of the dielectric permittivity step. Data are compared with the TM wave with he same value of the parallel component $k_{\|}$. For the TE wave, we see that the $\kappa_{1}$ component depends only slightly on the permittivity of the upper media. Components of wave vectors are expressed in units of $k_{0}=\omega / c$

\section{Conclusion}

We analyzed the propagation of the TE polarized surface wave along the negative permeability metamaterial surface and calculated the transmission, reflection and radiative losses due to the scattering of the surface wave at an interface between two dielectrics covering the metamaterial. The most important result is that the radiative losses due to the scattering are much smaller than that for the TM surface wave propagating along the metal-dielectric interface. Therefore, the TE polarized surface wave is a very good candidate for applications in the two-dimensional optics.

This work was supported by project APVV n. 51003505 and project VEGA 0633/09.

\section{References}

wave through the interface is always accompanied by the radiation of plane waves. The plane waves are necessary to balance the inhomogeneous electric and magnetic fields of the surface waves. To explain the origin of extremely small radiation losses of the TE surface wave, we plot in Fig. 5 the components of the wave vector for both TM and TE surface waves. The main difference between the two waves lies in the permittivity dependence of $\kappa_{d}$. Contrary to the TM wave, we find that $\kappa_{d}$ depends only weakly on the dielectric permittivity for the TE wave. Consequently, weak radiative fields are sufficient to correct the field discontinuity on both sides of the $x=0$ interface, so that radiative losses are small.

[1] A. V. Zayats, I. I. Smolyaninov and A. A. Maradudin, Phys. rep. 408, 131 (2005).

[2] G. I. Stegeman, A. A. Maradudin, T. S. Rahman, Phys. Rev. B 232376 (1981).

[3] R. F. Oulton, D. F. P. Pile, Y. Liu and X. Zhang, Phys. Rev. B 76, 035408 (2007)

[4] T. Váry, P. Markoš, in Metamaterials IV, Proceedings 7353 of SPIE Congress, Prague (2009).

[5] E. N. Economou, Phys. Rev. 182, 539 (1968).

[6] P. Markoš and C. M. Soukoulis, Wave Propagation: From Electrons to photonic Crystals and Left-handed Materials Princeton Univ. Press (2008).

[7] D. R. Smith et al., Phys. Rev. Lett. 84, 4184 (2000).

[8] R. Ruppin, Phys. Lett. A 277, 61 (2000); J. Phys.: Condens. Matt. 13, 1811 (2001). 